\title{
Engraftment of allotransplantated tumour cells in adult rag2 mutant Xenopus tropicalis
}

Dieter Tulkens (1,2), Dionysia Dimitrakopoulou (1), Tom Van Nieuwenhuysen (1), Marthe Boelens (1), Suzan Demuynck (1), Wendy Toussaint (1,3), David Creytens $(2,4)$, Pieter Van Vlierberghe $(2,5)$ \& Kris Vleminckx $(1,2)$

(1) Department of Biomedical Molecular Biology, Ghent University, Ghent, Belgium

(2) Cancer Research Institute Ghent (CRIG), Ghent, Belgium

(3) Laboratory of Myeloid Cell Ontogeny and Functional Specialization, VIB-UGent Center for inflammation Research, Ghent, Belgium

(4) Department of Pathology, Ghent University and Ghent University Hospital, Ghent, Belgium

(5) Department of Biomolecular Medicine, Ghent University, Ghent, Belgium

\section{Abstract}

Modelling human genetic diseases and cancer in lab animals has been greatly aided by the emergence of genetic engineering tools such as TALENs and CRISPR/Cas9. We have previously demonstrated the ease with which genetically engineered Xenopus models (GEXM) can be generated. This included the induction of autochthonous tumour formation by injection of early embryos with Cas9 recombinant protein loaded with sgRNAs targeting multiple tumour suppressor genes. What has been lacking so far is the possibility to propagate the induced cancers via transplantation. In this paper we describe the generation of a rag2 ${ }^{-/}$knock-out line in Xenopus tropicalis that is deficient in functional T-and B-cells. This line was validated by means of an allografting experiment with a primary $t p 53^{\%}$ donor tumour. In addition, we optimized available protocols for sub-lethal gamma irradiation of $X$. tropicalis froglets. Irradiated animals also allowed stable, albeit transient, engraftment of transplanted tp $53^{-\%}$ tumour cells. The novel $X$. tropicalis rag $\%$ line and the irradiated wild type froglets will further expand the experimental toolbox in this diploid amphibian, and help to establish it as a versatile and relevant model for exploring human cancer.

\section{Introduction}

The earliest transplantation of human primary tumour cells in mammalian hosts was described by Dr. Harry S. N. Greene (1938). Gradually during the last decades, tumour transplantation has been recognized as an indispensable tool in the cancer research field and has been successfully performed not only in mammalian species such as mice [reviewed by Sharkey \& Fogh (1984)] but also in nonmammalian vertebrates like zebrafish [reviewed by Gansner et al. (2017)]. Cancer immunoediting, and more specifically cancer immunosurveillance, is an important process that can severely hamper 
engraftment of tumours in immunocompetent hosts (Dunn et al., 2002). In order to escape from this phenomenon either inbred or immunodeficient animals are required, thus allowing stable tumour progression after transplantation. Researchers working with mice were able to generate, amongst others, the 'nude mice' (lacking the thymus and thus functional T-cells), the NOD-SCID and SCID-beige mice that are deficient in both the T- and B-cell pool, and finally the NSG or NOG mice that additionally lack functional NK cells (Yoshida, 2020). More recently zebrafish have joined the field. Several protocols and resources are available in this species to achieve stable engraftment of transplanted cells such as for example sub-lethal irradiation (Traver et al., 2004), the use of a rag2 ${ }^{\text {E450fs }}$ immunocompromised animals (Tang et al., 2014) and the use of syngeneic zebrafish lines, e.g. the CG1-strain (Smith et al., 2010). Furthermore, for xenograft experiments this species holds great promise as the transparent casper strain allowed the tracing and functional characterization of fluorescently labelled human

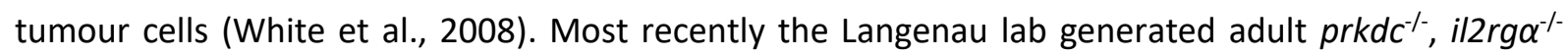
immunocompromised zebrafish in the casper-strain that allowed robust engraftment of human cancer cells (Yan et al., 2020).

Xenopus, like zebrafish enjoys transparency in embryonic stages, allowing tracing of fluorescently labelled cells. Besides, the Xenopus innate and adaptive immune cells and mechanisms show high conservation with their respective mammalian counterparts (Banach \& Robert, 2017). Despite the emergence of Xenopus tropicalis as a cancer model, thanks to the ease with which genetically engineered Xenopus models (GEXM) can be generated, so far experiments with tumour transplantations have not been documented for this species. Transplantations of $X$. laevis ff- 2 lymphoid tumour cells in inbred MHC homozygous ff $X$. laevis animals have led to the interesting finding that grafts are accepted in transplanted tadpoles but rejection is present in transplanted adults (Robert et al., 1995, 1997). This phenomenon is believed to be due to the second histogenesis present in the thymus during and after metamorphosis (Robert et al., 1995, 1997). Recently, Rollins-Smith \& Robert (2019) described a protocol to induce lymphocyte deficiency by subjecting $X$. laevis frogs to sub-lethal gamma irradiation. Another study (Rau et al., 2001) showed engraftment successes after transplanting the 15/0 lymphoid tumour line (from a spontaneous $X$. laevis thymoma) in $X$. laevis irradiated hosts. We describe here the generation and validation of a novel immunodeficient rag $^{-1-} X$. tropicalis line, suitable for transplantation experiments. Furthermore, we optimized and validated current available protocols for transplanting primary Xenopus tumours, for the first time, in irradiated $X$. tropicalis hosts. We believe these robust tools will be of high value for Xenopus tumour transplantation experiments and tumour immunity studies in general.

\section{Results}


In order to generate a $X$. tropicalis rag2 $\%$ line, an sgRNA was designed targeting the first fifth of the rag2 single exon gene. Wild type embryos were injected with a mixture of the selected sgRNA and Cas9 recombinant protein (Fig. 1A). To analyse editing efficiency, stage NF 41 embryos were lysed and genotyped. Amplicon deep sequencing (MiSeq ${ }^{\mathrm{TM}}$ System - Illumina) of the targeted region in the rag2 gene revealed a major inclusion of a 4 bp deletion, which is in correspondence with what is predicted by the inDelphi CRISPR repair outcome prediction algorithm (Shen et al., 2018). Correlation analysis revealed a significant high overall correlation between predicted and endogenously observed frequencies of variant calls (Pearson $r=0.9886, p<0.0001$ ) (Fig. 1B) confirming previous findings proposing inDelphi as suitable method for predicting CRISPR/Cas9 induced repair outcomes in $X$. tropicalis (Naert, Tulkens, et al., 2020). For obtaining homozygotes (see schematic Fig. 1A), first, crispant mosaic mutant animals were raised until adulthood, outcrossed with wild type animals and checked for germline transmission in the progeny. Heterozygote rag2 $^{+/ m u t}$ animals were subsequently intercrossed and homozygote rag $2^{\text {mut/mut }}$ animals were selected using a mixed Heteroduplex Mobility Assay (mHMA) genotyping technique (Foster et al., 2019) (Fig. 1C top). Sanger sequencing confirmed biallelic presence of a 4 bp deletion in homozygous mutant animals (Fig. 1C bottom). This deletion induces a frameshift after amino acid 91 resulting in a non-functional protein. Therefore, these animals are further referred to as $\mathrm{rag}_{2} \%$.

\section{Transplantation of $X$. tropicalis tp $53^{\%}$ tumour in an $X$. tropicalis rag2 ${ }^{-/}$adult}

To assess transplantation potential in the novel $\mathrm{rag}^{-} \%$ line, a thymic tumour originating from an adult tp53\% animal from a previous study (Naert, Dimitrakopoulou, et al., 2020) was isolated (Fig. 2A). Two parallel transplantations were performed: $5 \times 10^{6}$ tumour single cells were transplanted intraperitoneally (IP) in a rag $^{-\%}$ and a wild type adult as illustrated in Fig. 2B. Ten weeks post transplantation the rag $^{-\%}$ transplanted animal showed obvious signs of lethargy, while the transplanted wild type showed no signs of discomfort. A clear externally visible outgrowth was present in the rag2 ${ }^{-1}$ animal close to the transplantation injection site (Fig. 2C). Upon dissection multiple sites of engraftment were observed on the abdominal muscle wall and in the peritoneal cavity (Fig. 2D). Histopathological analysis of the tumours revealed presence of both epithelial and mesenchymal cell clusters, thereby showing morphological similarities to the donor tumour (Fig. 2E, top). Interestingly, multiple zones with neovascularization were present in these tumour engraftment sites (Fig. 2E, top). In addition, immunohistochemistry showed high proliferative capacity in both donor and engrafted tumours as indicated by PCNA immunostaining (Fig. 2E, bottom). Finally, the in-house developed mixed HMA method confirmed the inclusion of the same tp53 mutational variant, present in both the donor and the engrafted tumour (Fig. 2F). These data show that adult rag2 ${ }^{-1}$ knock-out $X$. tropicalis allows stable allografting of transplanted GEXM-derived tumour cells. 


\section{Transplantation validation in irradiated $X$. tropicalis animals}

Efficient tumour cell transplantation might also be achieved via alternative techniques apart from the generation of a rag $2 \%$ line. Immunocompromised $X$. laevis animals can also be obtained by sub-lethal gamma irradiation (Rollins-Smith \& Robert, 2019). In order to generate such hosts in X. tropicalis, an optimal dose suitable for successful allografting of tumour cells needed to be determined. We irradiated 3 different groups of 4-month-old froglets [ $8 \mathrm{~Gy}(\mathrm{n}=3), 10 \mathrm{~Gy}(\mathrm{n}=3)$ and $12 \mathrm{~Gy}(\mathrm{n}=3)$ ] and compared these with a non-irradiated wild type group ( $n=6$ ) (Fig. 3A). Approximately one week post irradiation all cohorts were euthanized and dissected. Major lymphoid organs (spleen and liver) and peripheral blood were checked to address irradiation potential. Natt and Herrick peripheral whole blood staining revealed significant reduction in white blood cell (WBC)/red blood cell (RBC) ratios in irradiated animals as compared to the non-irradiated controls $(p=0.0012)$ (Fig. 3B). Of note, no significant differences were present between the 3 irradiated groups. Furthermore, quantification of CD3 immunohistochemical stainings revealed that irradiation majorly impacted T-cell levels in both spleens and livers (Fig. 3C-D). For spleens, compared to the wild types (51.9\% \pm 4.5$)$, irradiation with an $8 \mathrm{~Gy}$ dose already induced a significant decrease in CD3 positivity $(36.0 \% \pm 5.9, p<0.05)$. This effect became more pronounced when irradiating to $10 \mathrm{~Gy}(15.7 \% \pm 2.1, p<0.001)$ and to $12 \mathrm{~Gy}(4.9 \% \pm 1.9$, $p<0.0001)$. Additionally, in the livers a similar dose-ratio trend was observed [wild type $(4.0 \% \pm 1.8)$, 8 Gy $(1.5 \% \pm 0.5, p=0.08), 10 \mathrm{~Gy}(0.4 \% \pm 0.1, p<0.05)$ and $12 \mathrm{~Gy}(0.2 \% \pm 0.1, p<0.05)]$. We propose irradiation up to a dose of $12 \mathrm{~Gy}$ is preferred for optimal reduction of T-cell numbers, thereby displaying the highest potential for successful tumour transplantation applications. In parallel with the experiment in $\mathrm{rag}^{-\%}$ animals, we validated the transplantation potential of $t p 53-$ mutant GEXM tumour cells also in an irradiated animal. For this purpose, an irradiated froglet (12 Gy) and a wild type sibling were injected intraperitoneally with $1 \times 10^{7}$ live tumour cells. To avoid any risk of repopulation of functional immune cells after the irradiation procedure, the froglets were analysed already 3 weeks post transplantation, in absence of any external signs indicative for engraftment. A clear increase of tumour cells circulating in the peritoneal cavity was observed in the irradiated transplant (non- $\mathrm{RBC} / \mathrm{RBC}=66.7 \% \pm 5.7$ ) as compared to the non-irradiated transplanted control (non$\mathrm{RBC} / \mathrm{RBC}=13.9 \% \pm 2.3$ ), where the non-RBC fraction (in the irradiated transplant) was majorly represented by tumour blast cells (Fig. 3E). Furthermore, in-depth histological analysis revealed tumour engraftment in both the kidney and the liver of the irradiated transplanted animal, whereas the wild type transplanted control did not show any signs of engraftment (Fig. 3F). Similar to what was found for the rag $\%$ animal, also tumour grafts observed in the irradiated transplant showed high proliferative capacity as indicated by PCNA immunostaining (Fig. 3G). 


\section{Discussion}

141 Donor cell rejection by the host organism after (allo)transplantation is a common hurdle, jeopardizing

142 bona fide assessment of engraftment potential of tumour cells. In absence of syngeneic models, the availability of immunocompromised animals is an absolute need to show evidence of engraftment after transplantation and to allow further phenotypic analysis of cancerous cells. We describe the generation of the novel $X$. tropicalis rag $2^{-\%}$ line as a beneficial tool for transplantation experiments. Due to the central role of the Rag2 protein in the process of $V(D) J$ recombination, these animals should lack mature T- and B-cells. Similar to what has been shown in zebrafish (Tang et al., 2014) also the $X$. tropicalis rag2 $\%$ animal used in this study allowed allografting of primary tumour donor cells injected intraperitoneally. Especially for longer incubations and serial tumour transplantations this line is recommended over irradiated animals where the transient nature of the immunosuppression might eventually hamper stable engraftment. Already 10 weeks post transplantation solid tumour grafts were visible at the injection site in the $\mathrm{rag}^{-} \%$ animal, whereas no signs of engraftment were observed in the control animal. Of note, previous transplantation studies with lymphosarcoma cells in X. laevis have shown how infectious Mycobacteria induced granulomas were mistakenly interpreted as the engrafted tumour cells (Asfari, 1988; Asfari \& Thiébaud, 1988). Therefore, we would like to state that validation of engraftment should not be solely based on histological assessment. In this study, for example, assessment of engraftment was done via endpoint histopathological analysis with an additional genotypic validation. Next to mutant or genetically modified hosts, the use of irradiated zebrafish (White et al., 2008) and mouse (Milas et al., 1987) animals have assisted greatly in the cancer research field. For Xenopus tropicalis limited data is available that show the potential of using this technique prior to performing allotransplantations. We showed that irradiating froglets with a dose of $12 \mathrm{~Gy}$, reduced T-cell numbers approximately 10 -fold in the spleen and 20-fold in the liver. We furthermore showed this dose allowed efficient engraftment of tp53\% tumour cells 3 weeks post intraperitoneal injection. Of note, using lower doses of radiation might also be sufficient to allow engraftment of host tumour cells. Goyos and colleagues (2011) showed that a $10 \mathrm{~Gy}$ irradiation dose already induced an inhibitory effect on thymocyte survival in $X$. tropicalis.

We hypothesize that engraftment success depends on multiple parameters such as tumour type, number of cells injected, injection site and incubation time in the host. Regarding the latter, it is known that repopulation of functional immune cells in irradiated animals can impair stable engraftment of tumour cells. For example, in zebrafish repopulation of myeloid, lymphoid an immune precursor cells

172 is observed already 2 weeks after irradiating adult zebrafish with 12 Gy (Traver et al., 2004). In 173 agreement with this finding, in another transplantation experiment with $X$. tropicalis GEXM tumour cells in 12 Gy irradiated hosts we indeed observed tumour cell clearance 5 weeks post transplantation, 
probably due to host immune cell repopulation (manuscript in preparation). Considering this caveat, the availability of the rag $^{-/}$line offers more flexibility with higher engraftment success rates even for long term experiments. We are convinced that with the generation of our novel rag2 ${ }^{-1}$ line - and the ease with which irradiation can be performed - studies on immune surveillance and tumour immunity will be significantly aided.

\section{Material and Methods}

\section{CRISPR/Cas9 mediated generation of mosaic mutant $X$. tropicalis animals}

The CRISPRScan software package (Moreno-Mateos et al., 2015) was used for the design of the rag2 CRISPR sgRNA. A 5'-gaattaatacgactcactataggGTCTTCCCTCCATGAATGgttttagagctagaaatagc-3' oligo along with the reverse oligo: aaaagcaccgactcggtgccactttttcaagttgataacggactagccttattttaacttgctatttctagctctaaaac-3' were ordered (Integrated DNA Technologies). At first DNA was prepared by annealing of the two primers and PCR amplification. The DNA template was in vitro transcribed using the HiScribe ${ }^{\mathrm{TM}}$ T7 High Yield RNA Synthesis Kit (New England Biolabs). The sgRNA was subsequently isolated using the phenolchloroform extraction/ $\mathrm{NH}_{4} \mathrm{OAc}$ precipitation method (Nakayama et al., 2014). RNA quantity was calculated by Qubit ${ }^{\circledR} 2.0$ Fluorometer (Thermo Fisher Scientific) measurement and quality was visually confirmed by agarose gel electrophoresis. A detailed guideline for generating the NLS-Cas9-NLS protein can be found in previous described work (Naert et al., 2016). After setting up natural matings resulting 2-cell stage embryos were injected unilaterally with a $1 \mathrm{nl}$ pre-incubated ( $30 \mathrm{sec} @ 37^{\circ} \mathrm{C}$ ) mix of sgRNA and Cas9 protein. Gene editing efficiencies were evaluated quantitatively by targeted amplicon next-generation sequencing (as described below). The inDelphi in silico prediction algorithm was included to validate endogenously observed frequencies of variant calls (Shen et al., 2018).

\section{DNA extraction and sequencing}

Gene editing was assessed by subjecting PCR amplified sgRNA targeted regions to deep sequencing followed by BATCH-GE analysis (Boel et al., 2016). DNA, from either whole embryos (three embryo pools each containing three stage NF 41 embryos) or from dissected tumours, was isolated using DNA lysis buffer (50 mM Tris pH 8.8, 1 mM EDTA, 0.5\% Tween- 20, $200 \mu \mathrm{g} / \mathrm{mL}$ proteinase $\mathrm{K}$ ) during an overnight incubation $\left(55^{\circ} \mathrm{C}\right)$ followed by a $5 \mathrm{~min}$ boiling step. Primers used in this study for

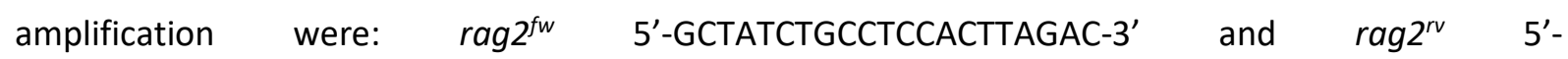
AATGTCAATGGTGTCATCATC- $3^{\prime}$ with an extra internal primer used for Sanger sequencing rag $2^{\text {int }} 5^{\prime}$ -

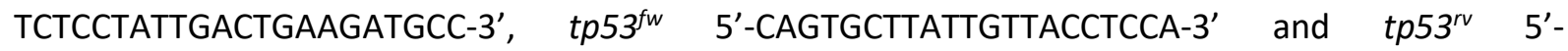
CATGGGAACTGTAGTCTATCAC-3'. The methodology for Sanger sequencing and correlation analysis 
between in vivo versus in vitro CRISPR mutational repair outcome can be found in (Naert, Tulkens, et al., 2020).

(Mixed) HMA genotyping method

For genotyping the rag2 line and tumour (graft) cells, WT DNA (i.e. DNA from non-injected frogs) was amplified in parallel with each unknown DNA sample via a standard PCR. Subsequently, equal quantities of both products - PCR amplified WT and unknown sample DNA - were mixed and eventually subjected to HMA in parallel with all the unknown samples individually (unmixed). This was completed by incubation of the samples at $98^{\circ} \mathrm{C}$ for 5 minutes, followed by a $4^{\circ} \mathrm{C}$ holding temperature using a transition with a ramp rate of $1^{\circ} \mathrm{C} / \mathrm{s}$. Finally, the PCR amplicons were prepped with DNA loading dye and run on an $8 \%$ (bis)acrylamide/TBE gel. Visualization was done on a Molecular Imager ${ }^{\circledR}$ Gel DocTM XR+ System (Bio-Rad) supported by the Image Lab software (Bio-Rad).

Irradiation procedure

24 hours prior transplantation, animals (early froglet stage) were sub-lethally irradiated up to $12 \mathrm{~Gy}$ with X-rays using the XRAD320 device (Precision X-Ray, Inc, North Branford, CT) at approximately 120 cGy/min. Froglets were placed individually in $50 \mathrm{~mL}$ Falcon tubes filled with $25 \mathrm{~mL}$ filter sterilised frog water.

\section{Tumour cell transplantation}

Tumour single cell suspensions were prepared manually by dissecting tumour pieces, subsequently washing them with sterile amphibian phosphate buffered saline (APBS) after which they were poured through a $40 \mu \mathrm{m}$ cell strainer (Falcon ${ }^{\mathrm{TM}}$ ) using tweezers to mince the tumour and APBS for flushing. An aliquoted $20 \mu \mathrm{L}$ of single cells was mixed with $180 \mu \mathrm{L} 0.1 \%$ tryphane blue solution to count living cells. Subsequently, the tumour cell suspension was centrifuged for $5 \mathrm{~min}$ at $240 \mathrm{~g}$ (RT) and resuspended with APBS to the appropriate concentration. Recipient host frogs ( $\mathrm{rag}^{-\%}$, irradiated or WT) were sedated using a $2 \mathrm{~g} / \mathrm{L} \mathrm{MS} 222$ (Tricaine methanesulfonate) solution diluted in water and adjusted to $\mathrm{pH}$ 7 with sodium bicarbonate. Each recipient host animal was injected intraperitoneally with a $100 \mu \mathrm{L}$ tumour single cell suspension containing $5 \times 10^{6}$ live tumour cells for the rag ${ }^{-\%}$ and respective adult control recipient and $1 \times 10^{7}$ live tumour cells for the irradiated and respective control froglet recipient, using BD Micro-Fine Demi $0.3 \mathrm{~mL}$ Syringes $0.3 \mathrm{~mm}$ (30G) x $8 \mathrm{~mm}$. Post transplantation, injected animals were housed separately and monitored closely for any signs of engraftment or discomfort. For all animal experiments, ethical approval was obtained and guidelines set out by the ethical committee were followed. 


\section{Blood counts}

Peripheral blood or intraperitoneal fluid was isolated by cardiac puncture or intraperitoneal (IP) lavage, respectively. For the IP lavage, a small incision was made in the skin of the belly and the abdominal muscle wall after which $100 \mu$ I APBS was used for rinsing the IP cavity. Approximately $10 \mu$ IP fluid cells diluted in APBS was collected for further processing. Immediately after collection, cells were diluted 1:50 in Natt and Herrick reagent, a methyl violet based staining solution, for downstream counting analysis (Maxham et al., 2016; Natt \& Herrick, 1952). Counts were performed using a Buerker hemocytometer (Marienfeld). For each Natt and Herrick sample at least 2x6 regions were counted (minimum 150 cells per count).

\section{Imaging, histology and immunohistochemistry}

Animals were euthanized by lethal incubation in a Benzocaine solution $(500 \mathrm{mg} / \mathrm{L})$ until heart beating stopped. Macroscopic images were taken with a Carl Zeiss StereoLUMAR.V12 stereomicroscope. Dissected organs or tumours were fixed overnight in $4 \%$ PFA at $4^{\circ} \mathrm{C}$ and subsequently dehydrated and paraffinized. Organ slices $(5 \mu \mathrm{m})$ were generated by microtomy and stained with haematoxylin and eosin using the Varistain ${ }^{\text {TM }}$ 24-4 Automatic Slide Stainer (Thermo-Scientific) for classical histological assessment. For immunohistochemistry (IHC) experiments following primary antibodies were used: IgG anti-human CD3 antibody (1:200, clone CD3-12, Bio-Rad) and anti-PCNA antibody (1:1000, PC10, Dako). Following secondary antibodies (all 1:500) were used: Biotinylated Goat Anti-Rat Ig (559286, BD Pharmingen) and Biotinylated Goat Anti-Mouse Ig (E0433, DAKO). DAB was used as chromogenic method of detection and signal was developed using the VECTASTAIN Elite ABC HRP Kit (PK-6100; Vector laboratories) combined with ImmPACT DAB Peroxidase (SK-4105; Vector laboratories). Finally, samples were counterstained with haematoxylin. All IHC experiments included 'no primary antibody' controls (data not shown). Imaging of sections was performed by using an Olympus BX51 Discussion Microscope. For quantification of CD3 stained slides, the QuPath software tool (Bankhead et al., 2017) was used. Slides were acquired using the ZEISS Axioscan 7 machine at 20x magnification with a resolution of $0.22 \mu \mathrm{m} /$ pixel.

\section{Statistical analysis}

Comparisons and conclusions between experimental and wild type groups were statistically supported by two-sided student's t-tests (non-significant $p \geq 0.05,{ }^{*} p<0.05,{ }^{* *} p<0.01,{ }^{* * *} p<0.001,{ }^{* * * *} p<$ 0.0001). Bar charts shown represent means with SD as error bar.

\section{Competing interests}

278 The authors declare no competing interests. 


\section{Contributions}

281 D.T., P.V.V. and K.V. designed the study. D.T., D.D., M.B., T.V.N. and S.D. were involved in the 282 generation and validation of the $\mathrm{rag}^{-\%}$ line. D.T. and W.T. performed the irradiation procedure. D.T. and K.V. performed all transplantations. D.C. performed histopathological validation of the tissue sections. D.T. \& K.V. wrote the manuscript.

\section{Acknowledgements}

287 D.T. holds a PhD fellowship from the Research Foundation-Flanders (FWO-Vlaanderen). Research in 288 the authors' laboratory is supported by the Research Foundation - Flanders (FWO-Vlaanderen) (grants G0A1515N and G029413N) and by the Concerted Research Actions from Ghent University (BOF15/GOA/011 and BOF20/GOA/023). Further support was obtained by the Hercules Foundation, Flanders (grant AUGE/11/14), the Desmoid Tumor Research Foundation, the Desmoid Tumour Foundation of Canada and SOS Desmoïde. In addition, the authors would like to thank Marjolein Carron and Annekatrien Boel for processing the next-generation sequencing data via the BATCH-GE software. We would like to acknowledge Amanda Gonçalves and Benjamin Pavie (VIB Bioimaging Core) for generating the QuPath script for positive cell detection. Furthermore, we are thankful to Tim Deceuninck for the good animal care. Finally, we would like to thank Joeri Tulkens for critical proofreading of the manuscript. 


\section{References}

Asfari, M. (1988). Mycobacterium-induced Infectious Granuloma in Xenopus: Histopathology and Transmissibility. Cancer Research, 48(4).

Asfari, M., \& Thiébaud, C. H. (1988). Transplantation Studies of a Putative Lymphosarcoma of Xenopus. Cancer Research, 48(4).

Banach, M., \& Robert, J. (2017). Tumor Immunology Viewed from Alternative Animal Models - the Xenopus Story. In Current Pathobiology Reports (Vol. 5, Issue 1, pp. 49-56). Springer. https://doi.org/10.1007/s40139-017-0125-y

Bankhead, P., Loughrey, M. B., Fernández, J. A., Dombrowski, Y., McArt, D. G., Dunne, P. D., McQuaid, S., Gray, R. T., Murray, L. J., Coleman, H. G., James, J. A., Salto-Tellez, M., \& Hamilton, P. W. (2017). QuPath: Open source software for digital pathology image analysis. Scientific Reports 2017 7:1, 7(1), 1-7. https://doi.org/10.1038/s41598-017-17204-5

Boel, A., Steyaert, W., De Rocker, N., Menten, B., Callewaert, B., De Paepe, A., Coucke, P., \& Willaert, A. (2016). BATCH-GE: Batch analysis of Next-Generation Sequencing data for genome editing assessment. Scientific Reports, 6(1), 30330. https://doi.org/10.1038/srep30330

Dunn, G. P., Bruce, A. T., Ikeda, H., Old, L. J., \& Schreiber, R. D. (2002). Cancer immunoediting: from immunosurveillance to tumor escape. Nature Immunology 2002 3:11, 3(11), 991-998. https://doi.org/10.1038/ni1102-991

Foster, S. D., Glover, S. R., Turner, A. N., Chatti, K., \& Challa, A. K. (2019). A mixing heteroduplex mobility assay (mHMA) to genotype homozygous mutants with small indels generated by CRISPR-Cas9 nucleases. MethodsX, 6, 1. https://doi.org/10.1016/J.MEX.2018.11.017

Gansner, J. M., Dang, M., Ammerman, M., \& Zon, L. I. (2017). Transplantation in zebrafish. Methods in Cell Biology, 138, 629-647. https://doi.org/10.1016/BS.MCB.2016.08.006

Goyos, A., Sowa, J., Ohta, Y., \& Robert, J. (2011). Remarkable Conservation of Distinct Nonclassical MHC Class I Lineages in Divergent Amphibian Species. The Journal of Immunology, 186(1), 372 381. https://doi.org/10.4049/JIMMUNOL.1001467

Greene, H. S. N. (1938). HETEROLOGOUS TRANSPLANTATION OF HUMAN AND OTHER MAMMALIAN TUMORS. Science, $88(2285)$.

Maxham, L. A., Forzán, M. J., Hogan, N. S., Vanderstichel, R. V., \& Gilroy, C. V. (2016). Hematologic reference intervals for Xenopus tropicalis with partial use of automatic counting methods and reliability of long-term stored samples. Veterinary Clinical Pathology, 45(2), 291-299. https://doi.org/10.1111/vcp.12362

Milas, L., Hunter, N., \& Peters, L. (1987). The tumor bed effect: dependence of tumor take, growth rate, and metastasis on the time interval between irradiation and tumor cell transplantation. 
International Journal of Radiation Oncology, Biology, Physics, 13(3), 379-383. https://doi.org/10.1016/0360-3016(87)90012-5

Moreno-Mateos, M. A., Vejnar, C. E., Beaudoin, J.-D., Fernandez, J. P., Mis, E. K., Khokha, M. K., \& Giraldez, A. J. (2015). CRISPRscan: designing highly efficient sgRNAs for CRISPR-Cas9 targeting in vivo. Nature Methods, 12(10), 982-988. https://doi.org/10.1038/nmeth.3543

Naert, T., Colpaert, R., Van Nieuwenhuysen, T., Dimitrakopoulou, D., Leoen, J., Haustraete, J., Boel, development in Xenopus tropicalis. Scientific Reports, 6(1), 35264. https://doi.org/10.1038/srep35264

Naert, T., Dimitrakopoulou, D., Tulkens, D., Demuynck, S., Carron, M., Noelanders, R., Eeckhout, L., Van Isterdael, G., Deforce, D., Vanhove, C., Van Dorpe, J., Creytens, D., \& Vleminckx, K. (2020). RBL1 (p107) functions as tumor suppressor in glioblastoma and small-cell pancreatic neuroendocrine carcinoma in Xenopus tropicalis. Oncogene 2020 39:13, 39(13), 2692-2706. https://doi.org/10.1038/s41388-020-1173-z

Naert, T., Tulkens, D., Edwards, N. A., Carron, M., Shaidani, N.-I., Wlizla, M., Boel, A., Demuynck, S., Horb, M. E., Coucke, P., Willaert, A., Zorn, A. M., \& Vleminckx, K. (2020). Maximizing CRISPR/Cas9 phenotype penetrance applying predictive modeling of editing outcomes in Xenopus and zebrafish embryos. Scientific Reports 2020 10:1, 10(1), 1-12. https://doi.org/10.1038/s41598-020-71412-0

Nakayama, T., Blitz, I. L., Fish, M. B., Odeleye, A. O., Manohar, S., Cho, K. W. Y., \& Grainger, R. M. (2014). Cas9-Based Genome Editing in Xenopus tropicalis. The Use of CRISPR/Cas9, ZFNs, TALENs in Generating Site Specific Genome Alterations, 546, 355-375. https://doi.org/10.1016/B978-0-12-801185-0.00017-9

Natt, M. P., \& Herrick, C. A. (1952). A New Blood Diluent for Counting the Erythrocytes and Leucocytes of the Chicken. Poultry Science, 31(4), 735-738. https://doi.org/10.3382/ps.0310735 perspective. Transplantation, 72(11), 1830-1835. https://doi.org/10.1097/00007890200112150-00020

Robert, J., Guiet, C., Cohen, N., \& Pasquier, L. Du. (1997). Effects of thymectomy and tolerance induction on tumor immunity in adult Xenopus laevis. International Journal of Cancer, 70(3), 330-334. https://doi.org/10.1002/(SICI)1097-0215(19970127)70:3<330::AID-IJC14>3.0.CO;2-J transplanted tumor in Xenopus laevis. Differentiation, 59(3), 135-144. https://doi.org/10.1046/j.1432-0436.1995.5930135.x 
Rollins-Smith, L. A., \& Robert, J. (2019). Lymphocyte Deficiency Induced by Sublethal Irradiation in Xenopus. Cold Spring Harbor Protocols, 2019(1), pdb.prot097626. https://doi.org/10.1101/PDB.PROT097626

Sharkey, F., \& Fogh, J. (1984). Considerations in the use of nude mice for cancer research. Cancer Metastasis Reviews, 3(4), 341-360. https://doi.org/10.1007/BF00051459

Shen, M. W., Arbab, M., Hsu, J. Y., Worstell, D., Culbertson, S. J., Krabbe, O., Cassa, C. A., Liu, D. R., Gifford, D. K., \& Sherwood, R. I. (2018). Predictable and precise template-free CRISPR editing of pathogenic variants. Nature 2018 563:7733, 563(7733), 646-651. https://doi.org/10.1038/s41586-018-0686-x

Smith, A. C. H., Raimondi, A. R., Salthouse, C. D., Ignatius, M. S., Blackburn, J. S., Mizgirev, I. V., Storer, N. Y., de Jong, J. L. O., Chen, A. T., Zhou, Y., Revskoy, S., Zon, L. I., \& Langenau, D. M. (2010). High-throughput cell transplantation establishes that tumor-initiating cells are abundant in zebrafish T-cell acute lymphoblastic leukemia. Blood, 115(16), 3296-3303. https://doi.org/10.1182/blood-2009-10-246488

Tang, Q., Abdelfattah, N. S., Blackburn, J. S., Moore, J. C., Martinez, S. A., Moore, F. E., Lobbardi, R., Tenente, I. M., Ignatius, M. S., Berman, J. N., Liwski, R. S., Houvras, Y., \& Langenau, D. M. (2014). Optimized cell transplantation using adult rag2 mutant zebrafsh. Nature Methods, 11(8), 821824. https://doi.org/10.1038/nmeth.3031

Traver, D., Winzeler, A., Stern, H. M., Mayhall, E. A., Langenau, D. M., Kutok, J. L., Look, A. T., \& Zon, L. I. (2004). Effects of lethal irradiation in zebrafish and rescue by hematopoietic cell transplantation. Blood, 104(5), 1298-1305. https://doi.org/10.1182/BLOOD-2004-01-0100

White, R. M., Sessa, A., Burke, C., Bowman, T., LeBlanc, J., Ceol, C., Bourque, C., Dovey, M., Goessling, W., Burns, C. E., \& Zon, L. I. (2008). Transparent adult zebrafish as a tool for in vivo transplantation analysis. Cell Stem Cell, 2(2), 183. https://doi.org/10.1016/J.STEM.2007.11.002

Yan, C., Do, D., Yang, Q., Brunson, D. C., JF, R., \& Langenau, D. M. (2020). Single cell imaging of human cancer xenografts using adult immune-deficient zebrafish. Nature Protocols, 15(9), 3105. https://doi.org/10.1038/S41596-020-0372-Y

Yoshida, G. J. (2020). Applications of patient-derived tumor xenograft models and tumor organoids. Journal of Hematology \& Oncology 2020 13:1, 13(1), 1-16. https://doi.org/10.1186/S13045019-0829-Z 
400

401

402

403

404

405

406

407

408

409

410

411

412

413
A

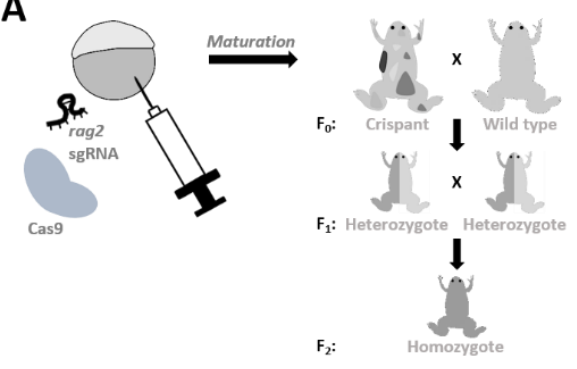

B

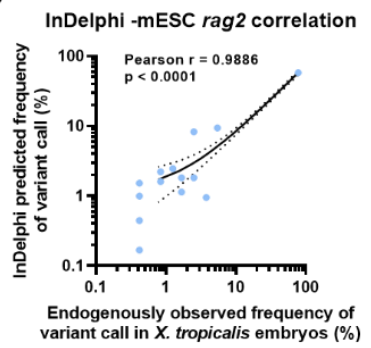

C

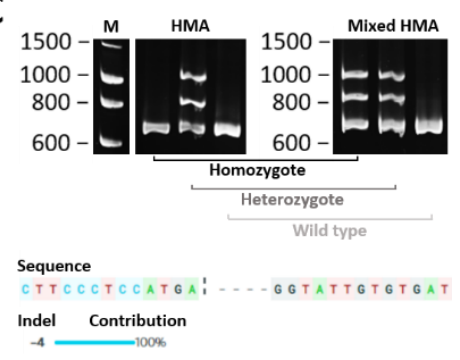

Figure 1. Generation of the $X$. tropicalis rag2 $\%$ knock-out line. (A) Embryos were injected with an sgRNA targeting the rag2 gene along with Cas9 protein. When sexually mature, animals were outcrossed to wild types to obtain heterozygous animals that were subsequently incrossed to obtain rag2 homozygous mutant animals in the $F_{2}$ generation. (B) Scatter plot showing correlation between in vivo observed mutational CRISPR repair outcomes in injected embryos ( $x$-axis) versus predicted outcomes using the inDelphi algorithm tool (y-axis). Dashed lines show the $95 \%$ confidence interval corresponding to the best-fit linear regression line (solid line). (C) Images taken from DNA electrophoresis gels after performing a normal HMA (left) and mixed HMA (right). Normal HMA included heating of the unknown PCR amplicons followed by slowly cooling and loading on the gel, while for mixed HMA, unknown PCR samples were first mixed with wild type rag2 amplicons after which the normal HMA was performed. Multiple bands present in both gels indicate heterozygous animals, while extra bands appearing after performing the mixed HMA (right gel) relate to homozygous mutant animals. Absence of any extra bands is indicative of wild type animals. 
A

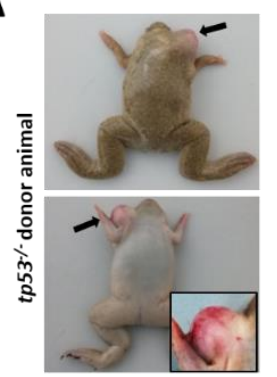

D

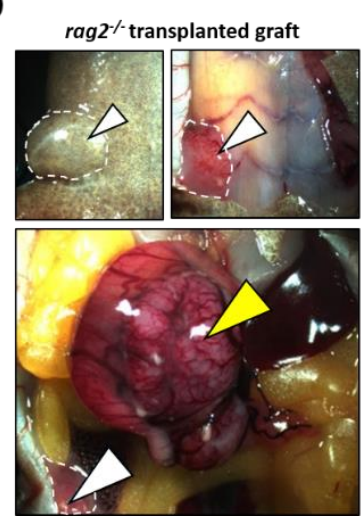

B

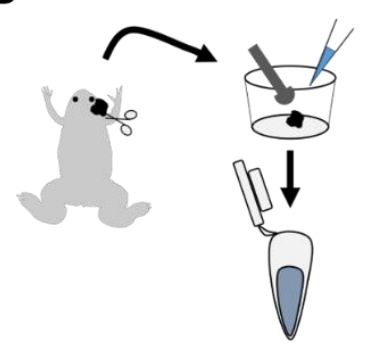

$\mathbf{E}$

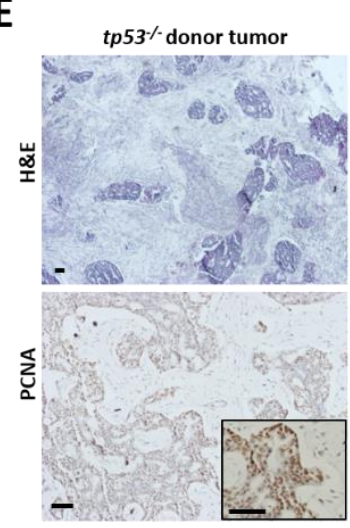

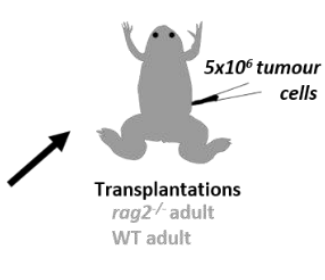

rag $\%$ transplanted graft

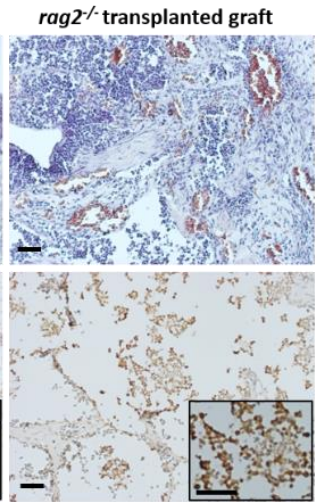

C

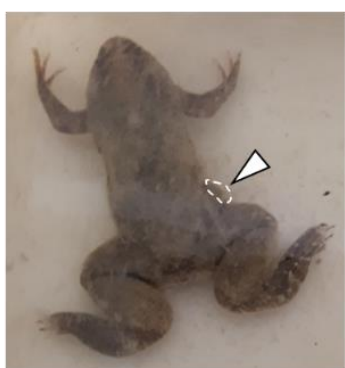

$\mathbf{F}$

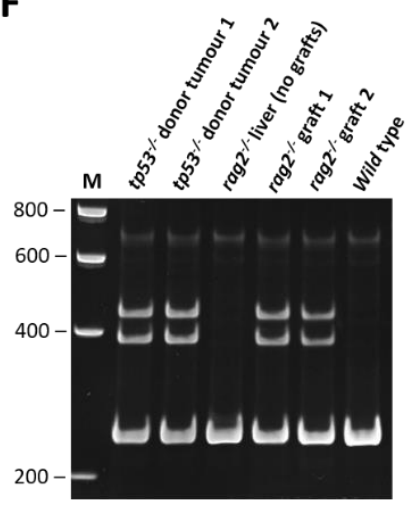

415

Figure 2. Validation of allografting in X. tropicalis rag $\%$ animals. (A) tp $53 \%$ donor animal harbouring a thymic tumour (black arrows). (B) Transplantation strategy including single cell generation using a 40 $\mu \mathrm{m}$ strainer followed by IP injections in a rag2 $\%$ adult and a wild type adult control (both $5 \times 10^{6}$ live cells). (C) A rag2 $\%$ transplanted animal with visual subcutaneous outgrowth close to the injection site (white arrow, white dashed line) 10 weeks post-transplantation. (D) Dissection microscopy images (ventral view) of rag $^{-\%}$ transplanted animal showing external (top left) and internal (top right \& bottom) views of the engrafted tumour at the injection site (white arrowheads, white dashed line) with an additional tumour mass in the intestinal mesenterium (yellow arrowhead). (E) H\&E and IHC stained sections from primary tumour in $t p 53^{-}$donor animal and the tumour graft in the $\mathrm{rag}^{-}$animal transplanted with the tp53\% tumour cells. (F) DNA electrophoresis gel image after performing a mixed HMA (for tp53 gene) on DNA from two tp53\% tumour samples (donor animal), liver (without grafts) and two tumour grafts obtained from the transplanted $\mathrm{rag}^{--}$animal and finally DNA from a tumour cell transplanted wild type animal. All scale bars are $50 \mu \mathrm{m}$. 
A

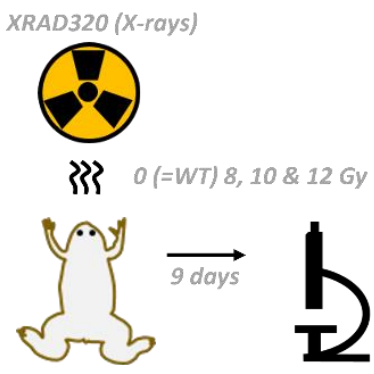

WT irradiated host

B

Cell counts

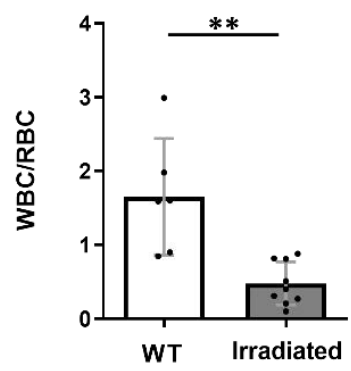

D Spleen $\mathrm{CD} 3$

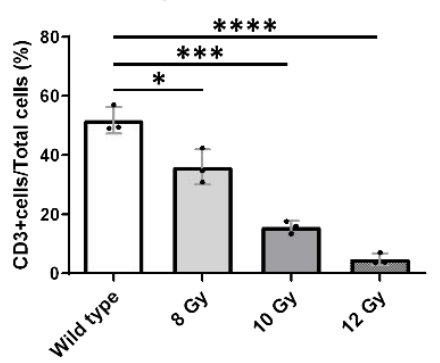

Liver CD3

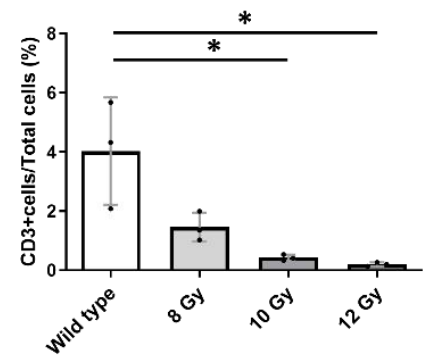

$\mathbf{E}$

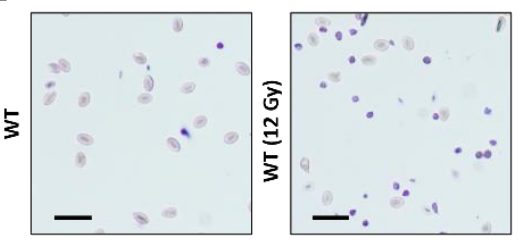

$\mathbf{F}$
C
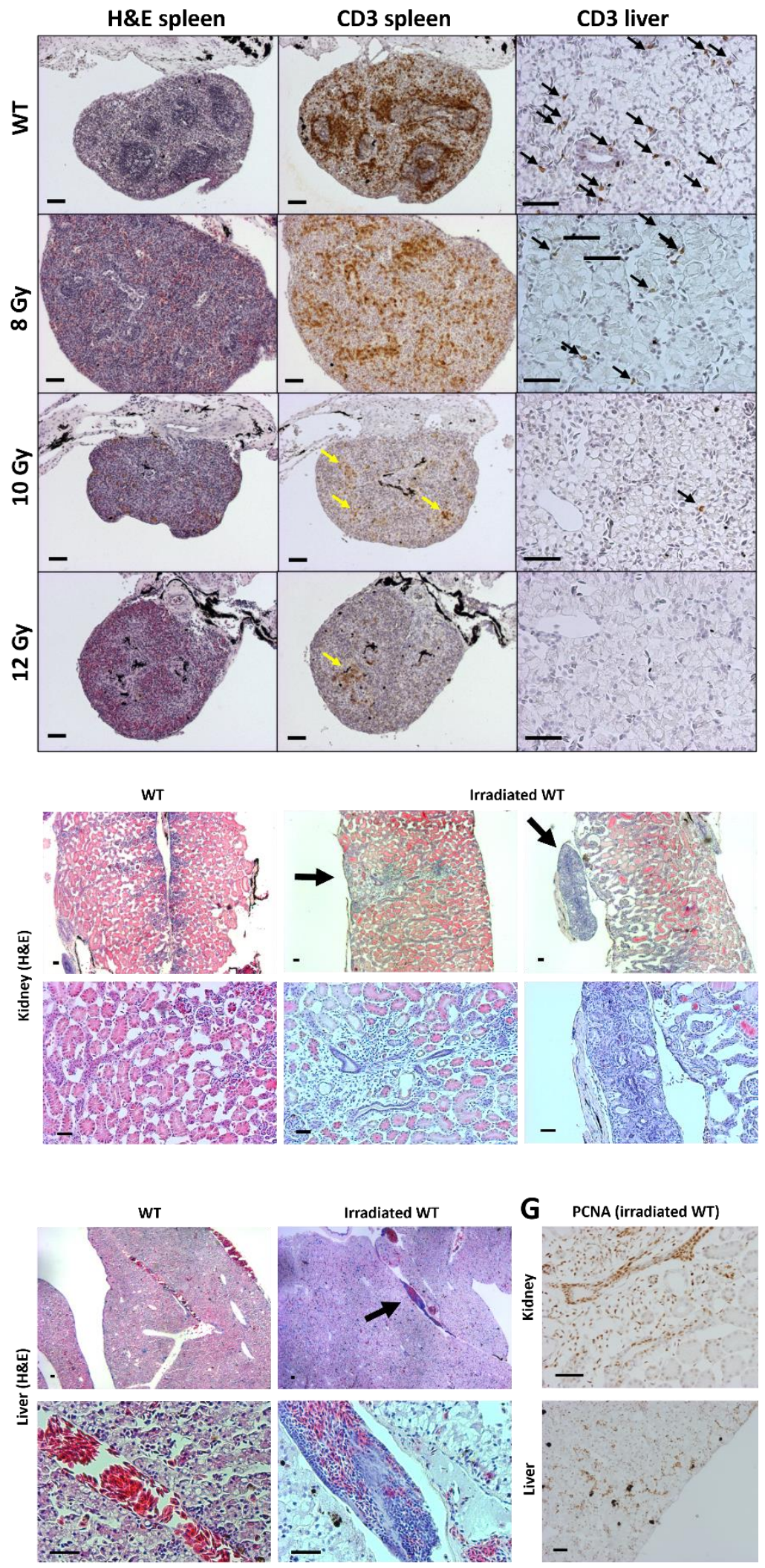

Figure 3. Allografting in irradiated wild type $X$. tropicalis animals. (A) Representation of the irradiation

procedure for which 3 groups (each $n=3$ ) were irradiated with $X$-rays (8, 10 and $12 \mathrm{~Gy}$ ) and compared by white blood cell (WBC)/red blood cell (RBC) ratios of irradiated animals and non-irradiated controls. 
435 (C) H\&E and anti-CD3 immunostained sections from spleens and livers of all 4 groups. Yellow arrows 436 show CD3 positive zones in the spleen, black arrows show CD3 positive cells in the liver. (D) IHC 437 quantified CD3 data of spleens and livers using the open source digital analysis tool QuPath (Bankhead et al., 2017). (E) IP fluid from transplanted irradiated animal and non-irradiated control stained with Natt and Herrick reagent. (F) H\&E Sections of engrafted regions in kidney and liver from transplanted irradiated froglet (black arrows) compared to respective kidney and liver sections in the transplanted

441 non-irradiated control froglet. (G) PCNA-stained sections from irradiated transplant showing kidney

442 and liver engraftment sites. All scale bars are $50 \mu \mathrm{m}$. Bar charts shown represent means with SD as 443 error bar. 